\title{
The Effect of Inhibitor Concentrations on the Chemical and Mechanical Properties of Bis-GMA-Based Resin Dental Composites
}

\author{
Sanjay Karunagaran ${ }^{1} \&$ Tien-Min G. $\mathrm{Chu}^{2}$ \\ ${ }^{1}$ Department of Prosthodontics, University of Tennessee Health Science Center College of Dentistry, Memphis, \\ Tennessee, USA \\ ${ }^{2}$ Department of Restorative Dentistry, Indiana University School of Dentistry, Indianapolis, Indiana, USA \\ Correspondence: Sanjay Karunagaran, BDS, DDS, MSD, Assistant Professor, Department of Prosthodontics, \\ Assistant Director, Dental Materials, University of Tennessee Health Science Center College of Dentistry, 875 \\ Union Avenue, Memphis, TN 38163, Canada. Tel: 1-901-448-6200. E-mail: skaruaga@uthsc.edu
}

Received: January 4, 2013 Accepted: February 25, 2013 Online Published: March 15, 2013

doi:10.5539/jmsr.v2n2p118 URL: http://dx.doi.org/10.5539/jmsr.v2n2p118

\begin{abstract}
Bis-GMA (Bisphenol A glycidyl methacrylate) based resin composites in dentistry require UV induced light polymerization to be used as restorative materials. This polymerization induces shrinkage stresses on command light polymerization, which can be clinically significant. To resolve this issue inhibitors such as Bisphenol-A-glycidyl dimethacrylate (BHT) have been introduced as an alternative method to try to decrease this polymerization shrinkage. However increases in inhibitor concentration whilst reducing polymerization shrinkage might induce an effect on the mechanical properties of the resin composite. In addition, the oral cavity is a fluid environment and can induce changes in the resin composite. The aim of this study was to try to determine if an increase in inhibitor concentration had an effect on the flexural strength and elastic modulus of resin composites after short and medium term aging immersion. These assessments were accomplished by looking at Flexural Strength and Elastic Modulus after short and medium term aging immersion. An experimental composite was prepared using a blend of Bisphenol A glycidyl dimethacrylate (Bis-GMA): Urethane Dimethacrylate (UDMA): Triethylene glycol dimethacrylate (TEGDMA) (1:1:1 weight ratio) with 70 $\mathrm{wt} \%$ silanized glass fillers. Four group concentrations of BHT were tested mechanically using flexural strength and elastic modulus. Based on this laboratory study, changes in BHT concentrations can induce and maintain high strength in resin composites.
\end{abstract}

Keywords: composite resin, BHT, flexural strength, elastic modulus

\section{Introduction}

Dental Composites also known as "white filling materials" typically consist of a resin based oligomer matrix such as Bisphenol A glycidyl methacrylate (Bis-GMA) or Urethane Dimethacrylate (UDMA), and inorganic fillers such as Silicone dioxide (Silica). The filler provides wear resistance and translucency whilst the resin helps bind the fillers together providing the dentist the ability to manipulate the material into the desired shape to fill the dental cavity until polymerized. Bisphenol A glycidyl methacrylate (Bis-GMA) is a reaction product of Bisphenol A and glycidyl ester methacrylate. The advantages of Bis-GMA over other monomers are less shrinkage, higher modulus, and reduced toxicity due to its lower volatility and diffusivity into tissues (Sideridou et al., 2002). In dentistry it is used in resin-based sealants, which serves to form a protective coating or a barrier that isolates pits and fissures to help prevent caries in children and adults. Bis-GMA is also used to construct provisional or temporary crowns and resin composite restorative materials that are esthetic and durable. Light-polymerized dental composites are now the most dominant restorative material used in the clinical practice of dentistry. The mode of polymerization of these resin composites involves using a high-intensity light that converts the monomer or the oligomer into a polymer and this photo-polymerization induces a transformation of the material from a viscous paste into a final solid product which is deemed esthetically and functionally sound. Following light irradiation, the free radical polymerization of the monomer is initiated and this is accompanied by a cross-linking of the molecule, which results in polymerization shrinkage. This contraction shrinkage can range from $0.2 \%-1.7 \%$ when considering linear contraction percentages, or from $1.2 \%-5.5 \%$ when considering volume percentage changes. The internal stresses created within the material can then be transferred to the 
tooth-resin interface as undesirable tensile and shear forces (Feilzer et al., 1990; Uno \& Asmussen, 1991; Meredith \& Setchell, 1997; Palin et al., 2005).

In order to release some of these unwanted stresses the resin composite will tend to plastically flow from free surfaces, causing additional areas of weakness (Anusavice, 2003). The regions of interfacial bonding will also serve as zones of stress relief (Braga \& Ferracane, 2002). If the contraction stress exceeds the bond strength at the interface, adhesive failure and marginal leakage would be caused (Uno \& Asmussen, 1991). If the bond strength is however strong enough to withstand failure at the interface, alternatively, stress can be generated and transmitted towards other parts of the tooth, resulting in cuspal deformation, fracture or microcracks within enamel and dentin (Venhoven et al., 1993).

To offset this problem, the concept of soft start polymerization was introduced, which aimed at reducing contraction stresses in resin composites, by applying a low intensity power density light source at first and then increasing it to a maximum power level (Katona \& Winkler, 1994), either by, stepped, pulsed or ramped curing.

However this reduction in contraction stress was accompanied by additional flaws. In particular these resin composites resulted in polymers which were significantly more susceptible to softening, especially in ethanol, than the resins which were cured by the original light curing methods. This increase in softness was attributed to a reduction in the number of cross links. There was also a decrease in hardness, degree of conversion as well as other physical properties (Asmussen \& Peutzfeldt, 2001).

In an attempt to find alternative methods to retard and control this rapid polymerization process, others have looked at trying to increase the inhibitor concentration within the resin system (Al-Shammari, 2007). Inhibitors such as Butylated hydroxytoluene (BHT), have been used in the past in commercial resin composites to prevent premature polymerization, usually in concentrations around $0.01 \%$ by weight (Anusavice, 2003). Bisphenol-A-glycidyl dimethacrylate has however not been studied as a mode of reducing polymerization shrinkage until recently.

A recent study looked at the concept of the effect of varying inhibitor concentrations on the polymerization characteristics of light-cured resin composites, and showed promising results. They concluded that using high levels of inhibitors were an effective method to slow down polymerization and reduce contraction stresses without compromising the degree of conversion. When considering methacrylate based resins, the viscous liquid undergoes a gradual transformation into a rigid material by radical polymerization that involves the $\mathrm{C}=\mathrm{C}$ of the methacrylate group (Al-Shammari, 2007). The extent of the transformation of this double bond into a single bond is called the "degree of conversion". Authors have shown that the higher the degree of conversion the higher the mechanical properties of the resin matrix (Calheiros et al., 2004). However this is accompanied by an increase in contraction stress. The goal is therefore to maximize the degree of conversion whilst trying to minimize the polymerization shrinkage of resin composites.

When considering BHT concentration authors have shown that at $0.05,0.2,0.5$ and $1.0 \mathrm{wt} \%$ BHT there are no significant difference in the degree of conversion (Al-Shammari, 2007). Studies have shown that around 6 percent residual monomer remains in BisGMA/TEGMA based resin composites which can leach out into the body (Braga, 2002). Authors have also concluded that this slow curing process did not show any negative effects on the flexural strength, elastic modulus, fracture toughness and cross-linking density of the resins (Bausch et al., 1982). This study however failed to mention the effects of these varying inhibitor concentrations on mechanical properties over time.

Our study aimed to study the effect of short and medium term aging immersion (48 hours, 1 month, 2 months and 4 months) of the varying concentrations $(0.01 \%, 0.6 \%, 1.00 \%$ and $1.40 \%)$ of Bisphenol-A-glycidyl dimethacrylate and determine if this immersion had an effect on the flexural strength and elastic modulus. Immersion is an important factor to consider as the oral cavity is a liquid environment and therefore prone to material changes than can affect the polymeric resin material. When resin composites are immersed in aqueous solutions, water absorption occurs between the polymer chains, promoting swelling. This swelling can cause a decrease in bond strength between the polymer chains. The resin filler matrix can also be attacked at the silane interface, thereby lowering the composite resin mechanical properties (Azillah et al., 1998).

\section{Experimental Method}

\subsection{Specimen Preparation}

For our flexural testing we prepared four concentrations of Bisphenol-A-glycidyl dimethacrylate at 0.01, 0.6, 1.0 and $1.4 \mathrm{wt} \%$ and made 10 samples per concentration for each of the medium and short term immersion times of $48 \mathrm{hrs}, 1$ month, 2 months and 4 months. 
The resin composite for this study was prepared as a mixture with a 1:1:1 weight ratio of Bis-GMA (Bisphenol-A-glycidyl dimethacrylate), UDMA (Urethane dimethacyylate) and TEGMA (triethyleneglycol dimethacrylate), obtained from (Sigma-Aldrich, St Louis, MO, USA). In order to make the resin light activating, $1.0 \mathrm{wt} \%$ champhorquinone (CQ, Sigma-Aldrich) and $0.5 \mathrm{wt} \%$ dimethylaminoethyl methacrylate (DMAEMA, Sigma-Aldrich) were added. The BHT was ground using a pestle and mortar to obtain a powder consistency. This was added to the resin component and the mixture was continuously stirred, heated and vibrated in order to obtain a homogenous mix. Each resin group was then loaded with $65 \mathrm{wt} \%$ silanized barium glass filler ( $0.7 \mathrm{um}$ Pentron Technologies, Wallingford, CT, USA) and $5 \mathrm{wt} \%$ silanized silica nanofiller $(0.04 \mathrm{um}$, Pentron Technologies). The total amount of glass fillers will contain $70 \mathrm{wt} \%$ of the total weight. The addition of the filler was accompanied by continuous mixing under vacuum so as not to incorporate air bubbles. The mixing WAS carried out in a small glass well covered in tin foil, in a dark room, so as not to activate the camphorquinone.

\subsection{Flexural Strength and Elastic Modulus}

The flexural strength and flexural modulus were determined using the three-point bending test as specified by the ISO specification 4049 for resin composite materials testing. A total of four BHT groups were prepared, at $0.01,0.6,1.0$ and $1.4 \mathrm{wt} \%$, with 10 samples per group. These samples were immersed in distilled water at four time points of aging immersion at, $48 \mathrm{hrs}, 1$ month, 2 months and 4 months and then tested for flexure and modulus. The ISO 4049 mode of fabrication is outlined below for both flexural and sorption and solubility testing.

To maintain the uniform nature of the specimen a stainless steel split mould was used to prepare the specimens according to the ISO 4049 dimensional specifications. A glass slide and Mylar strip was placed beneath the mould, and resin composite was then placed and condensed using metal spatula. The resin composite was overfilled and another Mylar strip and glass slide placed on top, and firmly pushed down so as to prevent the formation of air bubbles or porosities within the specimen. The specimens were light cured with four overlapping irradiation cycles of 60 seconds on both the top and bottom surfaces with an Optilux curing unit (Model: 401, Demetron Research Corporation, Danbury, CT USA) to maintain that all specimen were polymerized in the same direction and with the same time intervals. The power density of the curing unit was measured using a Cure Rite Radiometer (Dentsply Caulk, Milford, DE, USA) and recorded. A power density of $650 \mathrm{~mW} / \mathrm{cm}^{2}$ was established for the curing process, in order to maintain uniformity with the previous experimental studies. Periodic measurements were taken between the groups to check if the bulb needed to be changed, or the mirrors needed to be cleaned in order to maintain uniform photopolymerization.

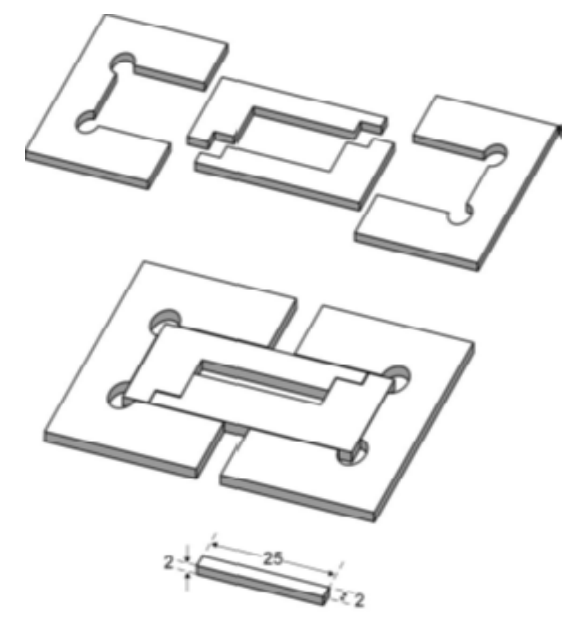

Figure 1. Stainless steel split mold for flexural strength specimen preparation

The specimens were then removed from the mould and the excess was removed, and then polished with SiC paper (600 grit), to achieve smooth edges. The final specimen's geometry was rectangular with dimensions of $25 \times 2 \times 2 \mathrm{~mm}^{3}$. They will then be stored in $37{ }^{\circ} \mathrm{C} \pm 1{ }^{\circ} \mathrm{C}$ PBS solution for 48 hours, 1 month, 2 month and 4 months. 
After each of the individual time points, the specimens were removed from the PBS solution, blotted dried, and subjected to the three point bending test. The tests were performed using a universal testing machine (Sintech Renew 1121, Instron Engineering Corp., Canton, MA, USA). A standard three point bending jig was attached to the machine and connected to a computer with a specifically designed program (Test-Works 3.0 MTS Systems Co., Eden Prairie, MN, USA). This software controlled the testing machine and recorded the breakage load and beam deflection. Before each test, the individual specimen thickness and width were recorded with a digital micrometer and introduced into the computer. The specimens were then placed on the jig and the test carried out using a crosshead speed of $1 \mathrm{~mm} / \mathrm{min}$ and a span length of $15 \mathrm{~mm}$. The Flexural strength and flexural modulus was calculated using the Equations (1) and (2) respectively:

$$
\begin{gathered}
\sigma_{b}=\frac{3 P L}{2 b h^{2}} \\
E_{b}=\frac{P^{\prime} L^{3}}{4 Y b h^{3}}
\end{gathered}
$$

$P=$ maximum breakage load $(\mathrm{N})$,

$\mathrm{L}=$ supporting span $(15 \mathrm{~mm})$,

$b=$ specimen width $(\mathrm{mm})$,

$h=$ specimen height $(\mathrm{mm})$.

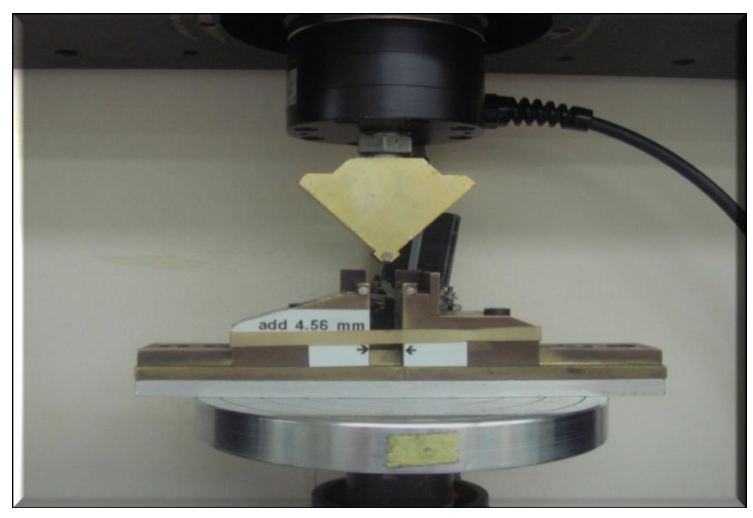

Figure 2. Three point bending apparatus

\subsection{Statistical Methods}

Statistical package SYSTAT (Craned Software International Ltd) was used. The effects of BHT concentration and time on flexural strength and elastic modulus were evaluated using two way analysis of variance (ANOVA) and Tukey's test.

\section{Results and Discussion}

Figure 3 shows the flexural strength at all concentrations of BHT after aging immersion. The statistical analysis was conducted by two ways ANOVA and Turkeys test for multiple comparisons. The flexural strength data for $0.01,0.6,1.0$ and $1.4 \mathrm{wt} \%$ BHT after aging immersion showed the following statistical results. 


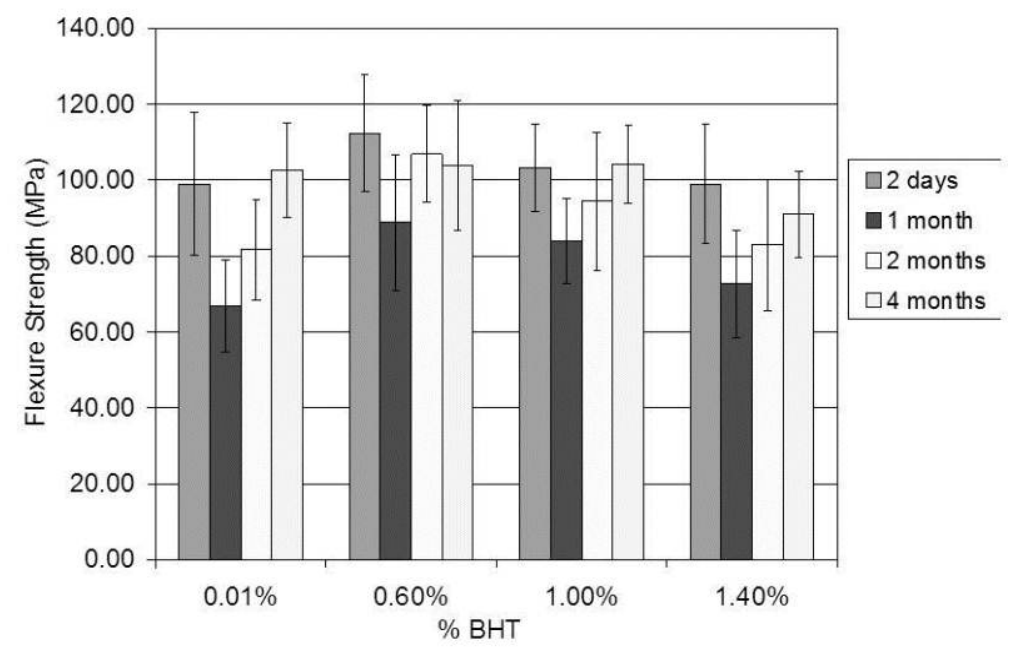

Figure 3. Wt $\%$ BHT versus flexural strength

There was no statistical difference between 2 days of immersion and 4 months of immersion $(p>0.05)$ at all concentrations of Bisphenol-A-glycidyl dimethacrylate.

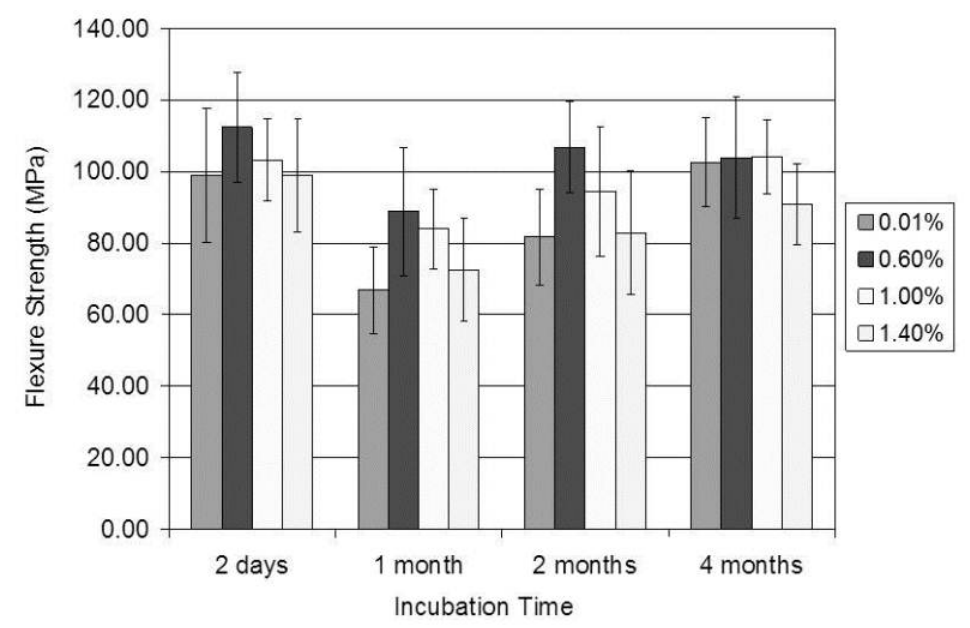

Figure 4. Incubation time versus flexural strength

Figure 4 compares flexural strength between the inhibitor concentrations at each of the individual aging immersion time points. The lowest flexural strength data was obtained at the 1 month time period for all groups which might be as a result of a unique process at this time point. There is a statistically significant $(p>0.05)$ difference in the flexural strength data between 0.01 and $1.4 \mathrm{wt} \%$ BHT.

Conclusion- for Flexural strength the interaction between BHT concentration and time aging was not significant $(\mathrm{p}=0.34)$, so the BHT concentration comparisons are similar regardless of time and the time comparisons are similar regardless of BHT concentration. BHT concentration had a significant effect on flexural strength $(\mathrm{p}=0.0001): 0.01 \%$ and $1.4 \%<1.0 \%<0.6 \%$. Aging immersion had a significant effect on flexural strength $(\mathrm{p}=0.0001): 0.01 \%$ and $1.40 \%>1.00 \%>0.6 \%$. 


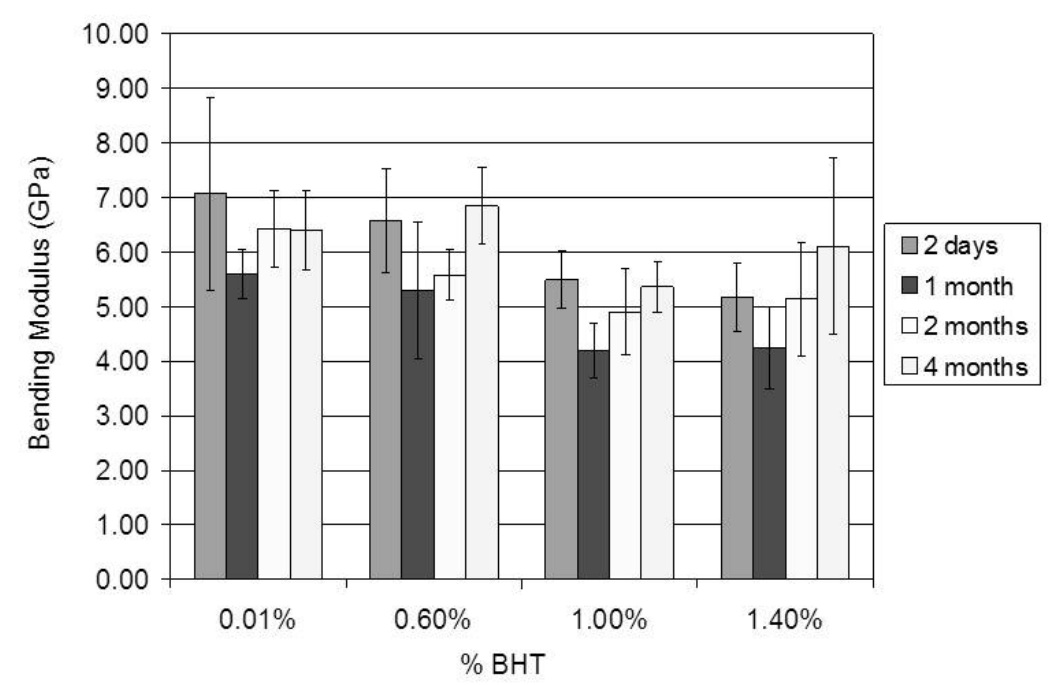

Figure $5 . \mathrm{Wt} \%$ BHT versus elastic modulus

Figure 5 shows flexural strength at all concentrations of BHT after aging immersion. The statistical analysis conducted by 2-way ANOVA and Turkeys for multiple comparisons produced the following conclusions. There is a general trend and statistical difference in modulus values between Groups 1 to 4 at each of the individual time points $(\mathrm{p}<0.05)$. There is a decrease in modulus values from 2 days to 4 months which is statistically significant $(\mathrm{p}<0.05)$. We concluded that BHT concentration affects elastic modulus at all aging immersion time points.

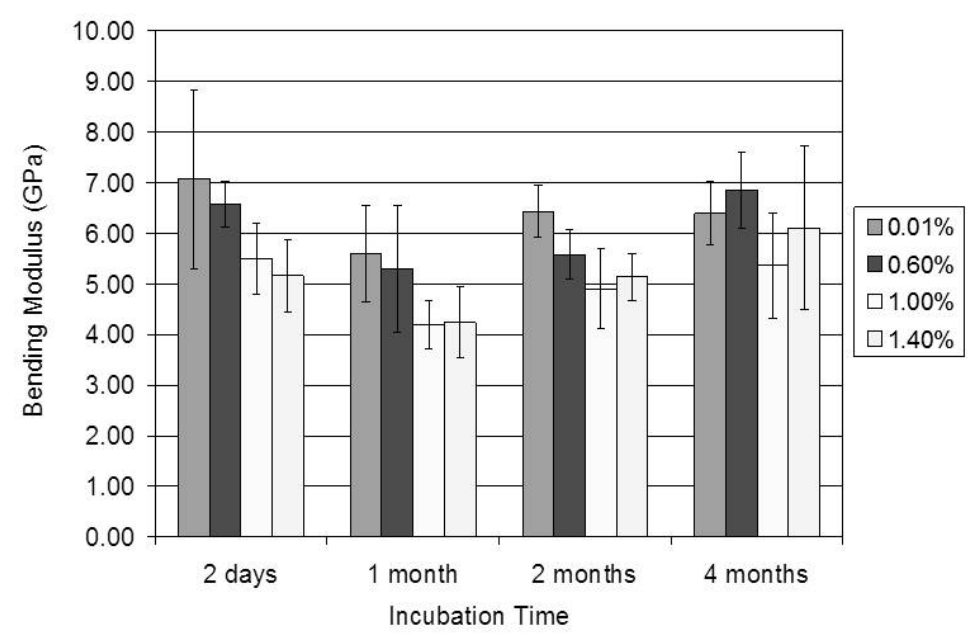

Figure 6. Incubation time versus Elastic Modulus

The statistical analysis conducted by 2-way ANOVA and Turkeys for multiple comparisons produced the following conclusions.

For Elastic Modulus the interaction between BHT concentration and time immersion aging was not significant $(\mathrm{p}=0.26)$, so the BHT concentration comparisons are similar regardless of time and the time comparisons are similar regardless of BHT concentration. BHT concentration had a significant effect on elastic modulus $(\mathrm{p}=0.0001): 0.01 \%$ and $0.6 \%>1.0 \%$ and $1.4 \%$. Time had a significant effect on elastic modulus $(\mathrm{p}=0.0001): 0.01 \%$ and $1.40 \%>1.00 \%>0.6 \%$. 
Authors have stated that short term immersion at 24 hours shows not significant reduction in flexural strength (Ferracane, 1994), which can also be concluded in our study. They also postulated that long term aging of 6 months has very little effect of flexural strength. Other authors have also reported that immersion in water for up to 6 months have shown a significant decrease in flexural strength. They concluded that this will depend on the type of resin composites, as water sorption may exert a degradative effect on the resin matrix and result in filler matrix debonding with a decrease in mechanical strength (Sideridou et al., 2003).

This study shows a progressive increase in flexural strength at 1,2 and 4 months. This may be attributed to an increase in polymerization of the methacrylate based monomers and unreacted monomers as they are incorporated into the polymer chain. In general once cyclization occurs with the formation of intramolecular bonds, a gel like state is formed and the unreacted carbon-carbon double bonds become trapped in the microgel which reduces the reactivity of the molecule and decreases the cross linking density. This may be a potential reason for the lower flexural strength values that are observed in this study at 1 month time periods. This has been shown to correspond to promote an increase in water sorption with an increase in the diffusion of water into these spaces. With time however there might be a decrease in the water sorption rate as materials are leached out enabling the remaining unreacted monomers to be able to react once again with the available carbon-carbon bonds and thereby increase the degree of conversion and thereby the flexural strength. It is know that the degree of conversion continues to increase with time.

\section{Conclusion}

In this study, the dental resin composite was synthesized in the composition that is common to materials on the market for clinical use. We however, varied the BHT inhibitor concentrations and the immersion time periods to study its effects on flexural strength and elastic modulus. The following conclusions were given.

1) BHT concentration had an effect on flexural strength: $0.01 \%$ and $1.40 \%<1.0 \%<0.6 \%$.

2) Aging immersion (time) had an effect on flexural strength: $0.01 \%$ and $1.40 \%>1.00 \%>0.6 \%$.

3) BHT concentration had an effect on elastic modulus: $0.01 \%$ and $0.6 \%>1.0 \%$ and $1.4 \%$.

4) Aging immersion (time) had an effect on elastic modulus: $0.01 \%$ and $1.40 \%>1.00 \%>0.6 \%$.

\section{References}

Al-Shammari, M. (2007). The effect of inhibitor concentration on polymerization characteristics of light-cured rein composites $(\mathrm{PhD})$. Indiana University School of Dentistry.

Anusavice, K. (2003). PHILLIPS' science of dental materials. Philadelphia, W. B. Saunders Company.

Asmussen, E., \& Peutzfeldt, A. (2001). Influence of pulse-delay curing on softening of polymer structures. $J$ Dent Res., 80(6), 1570-1573. http://dx.doi.org/10.1177/00220345010800061801

Azillah, M. A., Anstice, H. M., \& Pearson, G. J. (1998). Long-term flexural strength of three direct aesthetic restorative materials. $J$ Dent., 26(2), 177-182. http://dx.doi.org/10.1016/S0300-5712(96)00089-9

Bausch, J. R., de Lange, K., Davidson, C. L., Peters, A., de Gee, \& Anton J. (1982). Clinical significance of polymerization shrinkage of composite resins. $J$ Prosthet Dent, 48(1), 59-67. http://dx.doi.org/10.1016/0022-3913(82)90048-8

Braga, R. R., \& Ferracane, J. L. (2002). Contraction stress related to degree of conversion and reaction kinetics. J Dent Res, 81(2), 114-118. http://dx.doi.org/10.1177/154405910208100206

Calheiros, F. C., Braga, R. R., Kawano. Y., \& Ballester, R. Y. (2004). Relationship between contraction stress and degree of conversion in restorative composites. Dent Mater, 20(10), 939-946. http://dx.doi.org/10.1016/j.dental.2004.03.003

Feilzer, A. J., De Gee, A. J., \& Davidson, D. L. (1990). Quantitative determination of stress reduction by flow in composite restorations. Dent Mater, 6(3), 167-171. http://dx.doi.org/10.1016/0109-5641(90)90023-8

Ferracane, J. L. (1994). Elution of leachable components from composites. Journal of Oral Rehabilitation, 21, 441-452. http://dx.doi.org/10.1111/j.1365-2842.1994.tb01158.x

Katona, T. R., \& Winkler, M. M. (1994). Stress analysis of a bulk-filled Class V light-cured composite restoration. J Dent Res, 73(8), 1470-1477.

Meredith, N., \& Setchell, D. J. (1997). In vitro measurement of cuspal strain and displacement in composite restored teeth. J Dent, 25(3-4), 331-337. http://dx.doi.org/10.1016/S0300-5712(96)00047-4 
Palin, W. M., Fleming, G. J., Nathwani, H., Trevor-Burke, F. J., \& Randall, Ros C. (2005). In vitro cuspal deflection and microleakage of maxillary premolars restored with novel low-shrink dental composites. Dent Mater, 21(4), 324-335. http://dx.doi.org/10.1016/j.dental.2004.05.005

Sideridou, I., Tserki, V., \& Papanastasiou, G. (2002). Effect of chemical structure on degree of conversion in light-cured dimethacrylate-based dental resins. Biomaterials, 23(8), 1819-1829. http://dx.doi.org/10.1016/S0142-9612(01)00308-8

Sideridou, I., Tserki, V., \& Papanastasiou, G. (2003). Study of water sorption, solubility and modulus of elasticity of light-cured dimethacrylate-based dental resins. Biomaterials, 24(4), 655-665. http://dx.doi.org/10.1016/S0142-9612(02)00380-0

Uno, S., \& Asmussen, E. (1991). Marginal adaptation of a restorative resin polymerized at reduced rate. Scand $J$ Dent Res, 99(5), 440-444.

Venhoven, B. A., de Gee, A. J., \& Davidson, C. L. (1993). Polymerization contraction and conversion of light-curing BisGMA-based methacrylate resins. Biomaterials, 14(11), 871-875. http://dx.doi.org/10.1016/0142-9612(93)90010-Y 